\title{
Approximately Optimal Controllers for Quantitative Two-Phase Reach-Avoid Problems on Nonlinear Systems
}

\author{
Alexander Weber and Alexander Knoll
}

\begin{abstract}
The present work deals with quantitative two-phase reach-avoid problems on nonlinear control systems. This class of optimal control problem requires the plant's state to visit two (rather than one) target sets in succession while minimizing a prescribed cost functional. As we illustrate, the naive approach, which subdivides the problem into the two evident classical reach-avoid tasks, usually does not result in an optimal solution. In contrast, we prove that an optimal controller is obtained by consecutively solving two special quantitative reach-avoid problems. In addition, we present a fullyautomated method based on Symbolic Optimal Control to practically synthesize for the considered problem class approximately optimal controllers for sampled-data nonlinear plants. Experimental results on parcel delivery and on an aircraft routing mission confirm the practicality of our method.
\end{abstract}

\section{INTRODUCTION}

Cyber-physical systems have become a highly promising engineering technology which requires for its implementation expertise from many different disciplines like electrical and mechanical engineering, physics or computer sciences. This new concept to control a physical entity is based on feedback laws, which should be designed such that the physical process is controlled in a "reliable" and "efficient" way [1]. Reliability and efficiency for closed-loop systems are each a challenging requirement in itself.

Abstraction-based controller synthesis [2]-[5] has been developed to tackle the reliability requirement for feedback controllers on nonlinear systems. The said approach is a fullyautomated scheme for synthesizing controllers that come with formal guarantees to meet the specification. Symbolic Optimal Control [6]-[8] is a recent extension which aims at adding efficiency to the evolution of the closed loop within the meaning of minimizing a given cost functional.

Aforementioned synthesis approach contains two demanding steps with the first being the computation of a so-called discrete abstraction and the second being the solution of an auxiliary discrete problem. Though experts in this field proudly emphasize that complex control problems can be solved [3], [4] concrete algorithms (for performing the second step) have been presented so far only for a few classes of control problems. Most works investigate safety problems [9]-[11], classical reach-avoid problems [12]-[15] or reach-and-stay problems [16]. Some articles like [17]-[19] put emphasis on quantitative variants of previous problems, that is, additionally consider optimization of costs. So-called sequencing of targets, which requires the plant's state to visit $N$ target sets in a given order, is considered in [4] for the case $N=2$ and in [20], [21] for the case of integrator dynamics.

In this work, we consider a quantitative variant of sequencing for the case $N=2$, which we name quantitative two-phase reach-avoid problem. This specification requires the plant's state to visit two target sets in succession while minimizing a prescribed cost functional. (Obstacles should also be avoided.) In contrast to [4], [20], [21] we aim at solving the

The authors are with the Munich University of Applied Sciences, Dept. of Mechanical, Automotive and Aeronautical Eng., 80335 München, Germany. Corresponding author: A. Weber, weber13@hm. edu

This work has been supported by the German Federal Ministry of Education and Research (Project ARCUS). 


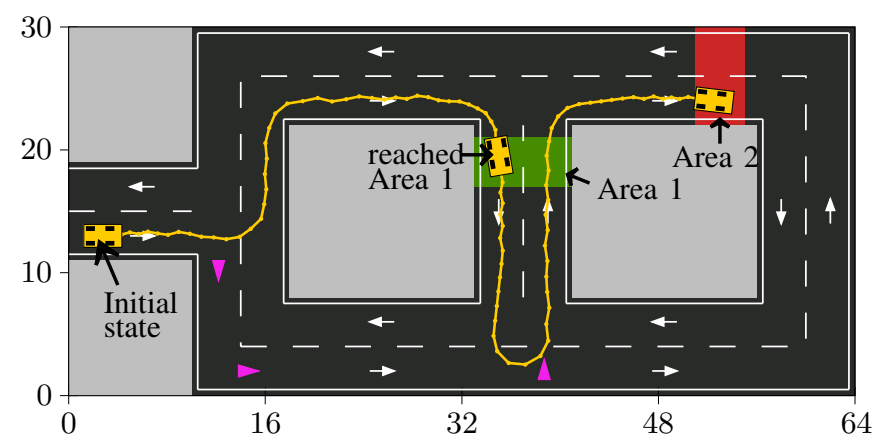

Fig. 1: Motivating example: A delivery vehicle has to make a delivery firstly in Area 1 and then in Area 2. A naive solution of this two-phase reach-avoid problem results in the highly inefficient trajectory that is depicted. Directions of the optimal trajectory are indicated by the purple arrows. All details to this example are given in Section IV-A.

problem optimally with respect to a rather arbitrary cost functional. An example of such a problem already appeared in the preceding work [15].

To give a concrete example at this point, consider Fig. 1: A delivery vehicle located in a Manhattan-like city has to make a delivery firstly in the green area ("Area 1") and then in the red area ("Area 2"). The question we address in this work is how to synthesize a controller fulfilling this specification and additionally operating for minimum cost in the sense of worst-case (or min-max) cost. The said problem could be naively solved by solving two ordinary (quantitative) reach-avoid problems as it was done in [4] for the special case of time-optimality. More concretely, the first problem is to steer the vehicle from Area 1 to Area 2. The second problem is to steer the vehicle from its initial state to the subset inside Area 1 on which the first controller is successful. Doing so, two controllers, each (approximately) optimal for the respective subproblem, have been synthesized, which steer the vehicle along the highly suboptimal path indicated in Fig. 1. The reason for the poor performance is that the manoeuvre to reach Area 1 optimally results in an orientation of the vehicle that is disadvantageous for reaching Area 2 next.

In this paper, we prove that an optimal controller can be synthesized through solving two special quantitative reach-avoid problems. Moreover, we present a fully-automated method based on aforementioned Symbolic Optimal Control to practically synthesize approximately optimal controllers on sampled versions of plants governed by the $n$-dimensional differential inclusion

$$
\dot{\xi}(t) \in f(\xi(t), u(t))+W .
$$

Here, $\xi$ is the $n$-dimensional state signal, $u$ is the input signal, $f$ is an ordinary function and $W$ is an $n$-dimensional set representing disturbances acting on the plant [4].

The paper is subsequently structured as follows. Basic concepts and definitions used throughout the paper are introduced in Section II. The main results on the synthesis of optimal controllers are given in Section III. In Section IV experimental results are presented and in particular the introductory example is investigated in all detail. Conclusions and open problems are discussed in Section V.

\section{PRELIMINARIES}

This work follows to a large extend the terminology of [7], [8]. For self-consistency, definitions used in this work are reviewed in this section, sometimes in an appropriately modified variant. More concretely, the following five subsections include basic notation, the concept of transition system and closed loop, the considered class of optimal control 
problems, the used notion of optimality and lastly a review of the classical reach-avoid problem formulation.

\section{A. Basic notation}

The empty set is denoted by $\emptyset . A \backslash B$ denotes the difference of two sets $A$ and $B$. The cardinality of a set $A$ will be denoted by $|A|$. The symbols $\mathbb{Z}, \mathbb{R}$ and $\mathbb{Z}_{+}, \mathbb{R}_{+}$stand for the set of integers and real numbers, respectively, and the respective subsets of non-negative elements. For $a, b \in \mathbb{R}$ the closed and half-open interval with endpoints $a$ and $b$ is denoted by $[a, b]$ and $[a, b[$, respectively. The symbols $[a ; b]$ and $[a ; b[$ denote the discrete intervals, e.g. $[a ; b]=[a, b] \cap \mathbb{Z}$. For a function $f: A \rightarrow B$ the restriction of $f$ to a subset $C$ of $A$ is denoted by $\left.f\right|_{C}[22$, p.4]. For $f: A \times B \rightarrow C$ and $b \in B$ the function $f(\cdot, b): A \rightarrow C$ maps $a \in A$ to $f(a, b)$. A set-valued map with domain $A$ and image the subsets of $B$ is denoted by $A \rightrightarrows B$ [23]. A set-valued map $f: A \rightrightarrows B$ is strict if $f(a) \neq \emptyset$ for all $a \in A$. The symbol $f \circ g$ stands for the composition of the maps $f$ and $g$. For sets $A, B$ the symbol $A^{B}$ stands for the set of all maps $B \rightarrow A$. An element of $A^{\mathbb{Z}_{+}}$is called signal. For $f \in A^{\mathbb{Z}_{+}}$and $\tau \in \mathbb{Z}_{+}$the symbol $\sigma^{\tau} f$ stands for the backwards shifted signal $g \in A^{\mathbb{Z}_{+}}$defined by $g(t)=f(t+\tau)$.

\section{B. System, controller and closed loop}

Let $X$ and $U$ be non-empty sets. A (transition) system with state space $X$ and input space $U$ is a triple

$$
(X, U, F) \text {, }
$$

where $F: X \times U \rightrightarrows X$ is a strict set-valued map (called transition function). System (2) is implicitly endowed with time-discrete dynamics implied by the difference inclusion

$$
x(t+1) \in F(x(t), u(t)) .
$$

The behaviour of a system $S$ of the form (2) initialized at $p \in X$ is the set of all signal pairs $(u, x) \in(U \times X)^{\mathbb{Z}_{+}}$such that $x(0)=p$ and (3) holds for all $t \in \mathbb{Z}_{+}$. This set is denoted by $\mathcal{B}_{p}(S)$.

The concept of a closed loop is formalized by means of a controller and the respective closed-loop behaviour: A controller is a strict set-valued map [7, Eq. 4]

$$
\mu: \bigcup_{T \in \mathbb{Z}_{+}}\left(X^{[0 ; T]} \times U^{[0 ; T[}\right) \rightrightarrows U \times\{0,1\},
$$

where the second component of the image reports whether the controller is in operation (' 0 ') or has stopped operating ('1') [7, Sec. III.A]. The set of all such maps is denoted by $\mathcal{F}(X, U)$. A controller $\mu \in \mathcal{F}(X, U)$ is called memoryless if a representation as a map $\mu: X \rightrightarrows U \times\{0,1\}$ is possible. The subset of memoryless controllers is denoted by $\mathcal{F}_{0}(X, U)$.

The closed-loop behaviour of a system $S$ of the form (2) interconnected with $\mu \in \mathcal{F}(X, U)$ and initialized at $p \in X$ is the set of all signals $(u, v, x)$ that satisfy $(u, x) \in \mathcal{B}_{p}(S)$ and

$$
(u(t), v(t)) \in \mu\left(\left.x\right|_{[0 ; t]},\left.u\right|_{[0 ; t]}\right)
$$

for all $t \in \mathbb{Z}_{+}$. The symbol $\mathcal{B}_{p}(\mu \times S)$ stands for this set. 


\section{Optimal control problem}

As explained later, this work considers slightly more general optimal control problems than formulated in [8]. Common with [8] is that the closed loop is leavable, i.e. the controller may stop its operation at any time. Moreover, stopping will be mandatory. Also, optimality is meant in terms of an accumulation of two kinds of costs that accrue for the operation of the closed loop. Costs may be infinite, which allows to formulate hard state and input constraints. Firstly, as usually in optimal control, there is a running cost

$$
g: X \times X \times U \rightarrow \mathbb{R}_{+} \cup\{\infty\}
$$

accruing between two consecutive points in time. Secondly, the closed-loop trajectory is rated once at stopping time by a trajectory cost

$$
G: \bigcup_{T \in \mathbb{Z}_{+}} X^{[0 ; T]} \rightarrow \mathbb{R}_{+} \cup\{\infty\}
$$

Altogether, for a signal pair $(u, x) \in \mathcal{B}_{p}(S)$ and a stopping signal $v \in\{0,1\}^{\mathbb{Z}_{+}}$we assign a total cost

$$
J:(U \times\{0,1\} \times X)^{\mathbb{Z}_{+}} \rightarrow \mathbb{R}_{+} \cup\{\infty\}
$$

defined by

$$
J(u, v, x)=\sum_{t=0}^{T-1} g(x(t), x(t+1), u(t))+G\left(\left.x\right|_{[0 ; T]}\right)
$$

for $T=\min v^{-1}(1)$ if $v \neq 0$, and $J(u, v, x)=\infty$ if $v=0$. (Note that the last case formalizes the requirement for stopping, which occurs at the first 0-1 edge in $v$.)

By putting together the previous objects we arrive at the subsequent definition.

II.1 Definition. Let $(X, U, F)$ be a system, and $G$ and $g$ be as in (5) and (4), respectively. The tuple

$$
(X, U, F, G, g)
$$

is called optimal control problem.

Definition II.1 agrees with [8, Def. III.3] if the trajectory cost in (5) possesses the special form

$$
G: X \rightarrow \mathbb{R}_{+} \cup\{\infty\} .
$$

In this case, the map in (8) is called terminal cost [8].

\section{Solution of an optimal control problem}

In order to introduce the notion of solution of an optimal control problem we introduce the following quantity [8].

II.2 Definition. Let $\Pi$ be an optimal control problem of the form (7) and let $J$ be as in (6). The closed-loop performance of $\Pi$ is the map assigning $(p, \mu) \in X \times \mathcal{F}(X, U)$ to

$$
\sup _{(u, v, x) \in \mathcal{B}_{p}(\mu \times S)} J(u, v, x),
$$

where $S=(X, U, F)$.

Having introduced the quantity (9) the concept of value function can be formulated as follows [8, Sec. III.A,VI.C]. 
II.3 Definition. Let $\Pi$ be an optimal control problem of the form (7). Denote by $L(p, \mu)$ the value (9). The value function of $\Pi$ is the map $V: X \rightarrow \mathbb{R}_{+} \cup\{\infty\}$ defined by

$$
V(p)=\inf _{\mu \in \mathcal{F}(X, U)} L(p, \mu) .
$$

A controller $\mu \in \mathcal{F}(X, U)$ is optimal for $\Pi$ if $V=L(\cdot, \mu)$. Such a pair $(V, \mu)$ is called solution of $\Pi$.

So, an optimal controller minimizes the worst-case costs.

\section{E. The classical reach-avoid problem}

The classical (qualitative) reach-avoid problem is the problem of steering the state signal of a system $S$ of the form (2) into a target set $A \subseteq X$ while avoiding an obstacle set $O \subseteq X$. This specification can be easily formulated as an optimal control problem (7), cf. [8, Ex. III.5]: Define the trajectory cost $G$ as a terminal cost (8) by

$$
G(p)= \begin{cases}0, & \text { if } p \in A \\ \infty, & \text { otherwise }\end{cases}
$$

and define the running cost $g$ in (4) by

$$
g(x, y, u)=\left\{\begin{array}{ll}
0, & \text { if } x \in X \backslash O \\
\infty, & \text { otherwise }
\end{array} .\right.
$$

An optimal controller is "successful" for every state signal that is initialized at a point in $X \backslash V^{-1}(\infty)$, where $V$ stands for the value function of the defined optimal control problem.

Clearly, a quantitative version of a reach-avoid problem is obtained when allowing $G$ and $g$ to be non-constant instead of 0 on the respective subsets. (Examples can be found in [15], [18].) We formalize this version in the next definition, which already indicates that for the upcoming contributions the key object will be the trajectory cost (rather than the running cost) of an optimal control problem.

II.4 Definition. Let $\Pi$ be of the form (7) such that $G$ satisfies (11) with $G_{0}(p)$ in place of 0 , where $G_{0}: X \rightarrow \mathbb{R}_{+} \cup\{\infty\}$ and $A \subseteq X$ is non-empty. Then $\Pi$ is called (quantitative) reach-avoid problem associated with $A$ and $G_{0}$.

We extend in this work Definition II.4 to a class of problems that contains an additional intermediate goal - a "stopover set", as explained below.

\section{QUANTITATIVE 2-PHASE REACH-AVOID PROBLEMS}

We divide the presentation of our main contributions into three parts. Two-phase reach-avoid problems are rigorously defined in Section III-A. In Section III-B we deduce the structure of an optimal controller for this class of problems. A constructive method to synthesize approximately optimal controllers on sampled-data systems is given in Section III-C.

\section{A. Problem definition}

We are going to investigate the following class of optimal control problem.

III.1 Definition. Let $\Pi$ be an optimal control problem of the form (7) such that $G$ is defined by

$$
G\left(\left.x\right|_{[0 ; t]}\right)= \begin{cases}G_{0}(x(t)), & \text { if } \exists_{s \leq t}: x(s) \in A_{1} \wedge x(t) \in A_{2} \\ \infty, & \text { otherwise }\end{cases}
$$


where $G_{0}: X \rightarrow \mathbb{R}_{+} \cup\{\infty\}$ and $A_{1}, A_{2} \subseteq X$ are non-empty sets. Then $\Pi$ is called (quantitative) two-phase reach-avoid problem associated with $A_{1}, A_{2}$ and $G_{0}$.

Thus, for a two-phase reach-avoid problem stopping may occur only if firstly $A_{1}$ is visited and then $A_{2}$ is reached.

\section{B. Structure of an optimal controller}

Our first result below defines a controller for a two-phase reach-avoid problem whose closed-loop performance is equal to the one of a (memoryless) controller for a special classical reach-avoid problem. The defined controller structure will turn out to be appropriate for the desired optimal controller.

III.1 Proposition. Let $\Pi$ be a two-phase reach-avoid problem of the form (7) associated with $A_{1}, A_{2}$ and $G_{0}$. Let $\Pi_{2}=\left(X, U, F, G_{2}, g\right)$ be the reach-avoid problem associated with $A_{2}$ and $G_{0}$. Let $\mu_{2} \in \mathcal{F}_{0}(X, U)$ and let $\Pi_{1}=\left(X, U, F, G_{1}, g\right)$ be the reach-avoid problem associated with $A_{1}$ and $L_{2}\left(\cdot, \mu_{2}\right)$, where $L_{2}$ denotes the closed-loop performance of $\Pi_{2}$. Let $\mu_{1} \in \mathcal{F}_{0}(X, U)$ and consider $\mu \in \mathcal{F}(X, U)$ defined by

$$
\mu\left(\left.x\right|_{[0 ; t]},\left.u\right|_{[0 ; t]}\right)=\left\{\begin{array}{ll}
\mu_{1}(x(t)), & \text { if } w([0 ; t])=\{0\} \\
\mu_{2}(x(t)), & \text { otherwise }
\end{array},\right.
$$

where $w:=\left(\mu_{1} \circ x\right)_{2}$ is the stopping signal of $\mu_{1}$. Then

$$
L(p, \mu)=L_{1}\left(p, \mu_{1}\right)
$$

for all $p \in X$, where $L$ and $L_{1}$ denote the closed-loop performance of $\Pi$ and $\Pi_{1}$, respectively.

By virtue of previous proposition we will be able to conclude the following central theorem of this work:

III.1 Theorem. Let $\Pi, \Pi_{1}, \Pi_{2}$ and $\mu, \mu_{1}, \mu_{2}$ be as in Proposition III.1. Additionally, assume that $\mu_{i}$ is optimal for $\Pi_{i}$ for $i \in\{1,2\}$. Then $\mu$ is optimal for $\Pi$.

Proof of Proposition III.1. Let $p \in X$. Denote by $J, J_{1}, J_{2}$ the total cost (6) as defined for $\Pi, \Pi_{1}, \Pi_{2}$, respectively.

Firstly, we prove that for all signals $(u, v, x) \in \mathcal{B}_{p}(\mu \times S)$, where $S=(X, U, F)$, it holds

$$
J(u, v, x) \leq L_{1}\left(p, \mu_{1}\right)
$$

if $L_{1}\left(p, \mu_{1}\right)$ is finite. Under the latter assumption, $\tau:=\inf w^{-1}(1)$ and $T:=\inf v^{-1}(1)$ are finite, where $w:=\left(\mu_{1} \circ x\right)_{2}$. Then, using (6b) as defined for $\Pi$ and $\Pi_{2}$ yields

$$
\begin{aligned}
J(u, v, x) & =\sum_{t=0}^{\tau-1} g(x(t), x(t+1), u(t))+\sum_{t=\tau}^{T-1} g(\ldots)+G_{0}(x(T)) \\
& =\sum_{t=0}^{\tau-1} g(x(t), x(t+1), u(t))+J_{2}\left(\sigma^{\tau} u, \sigma^{\tau} v, \sigma^{\tau} x\right) .
\end{aligned}
$$

(Above, the ellipsis stands for the evident arguments to g.) Using (9) as defined for $\Pi_{2}$ we see that the last term in the last sum is not greater than $L_{2}\left(x(\tau), \mu_{2}\right)$, so we conclude (14) using (6b) and (9) as defined for $\Pi_{1}$.

Next, assume $L(p, \mu)=\infty$. Then, for every $M \geq 0$ there exists a signal $(u, v, x) \in \mathcal{B}_{p}(\mu \times S)$ such that $J(u, v, x) \geq M$. Assume that, however, $L_{1}\left(p, \mu_{1}\right)$ is finite. Then, by (14), $M \leq$ $L_{1}\left(p, \mu_{1}\right)$, a contradiction. So, $L(p, \mu)=L_{1}(p, \mu)$.

Now, assume $L(p, \mu)<\infty$. Obviously, $L_{1}\left(p, \mu_{1}\right)<\infty$. Then, for $\varepsilon>0$ there exists $(u, v, x) \in$ 
$\mathcal{B}_{p}(\mu \times S)$ such that $L(p, \mu)<J(u, v, x)+\varepsilon<L_{1}\left(p, \mu_{1}\right)+\varepsilon$ by (14). The limit $\varepsilon \rightarrow 0$ implies $L(p, \mu) \leq L_{1}\left(p, \mu_{1}\right)$.

For the reverse inequality, let $\varepsilon>0$ and note that there exist $v \in\{0,1\}^{\mathbb{Z}_{+}}$and $(u, x) \in \mathcal{B}_{p}(S)$ such that the inequalities

$$
\begin{aligned}
& L_{1}\left(p, \mu_{1}\right)-J_{1}(u, v, x)<\varepsilon, \\
& L_{2}\left(x(\tau), \mu_{2}\right)-J_{2}\left(\sigma^{\tau} u, \sigma^{\tau} v, \sigma^{\tau} x\right)<\varepsilon
\end{aligned}
$$

hold, where $\tau:=\min v^{-1}(1)$. It follows that

$$
\begin{aligned}
& L_{1}\left(p, \mu_{1}\right)-J(u, v, x)<\varepsilon+J_{1}(u, v, x)-J(u, v, x)= \\
& =\varepsilon+L_{2}\left(x(\tau), \mu_{2}\right)-J_{2}\left(\sigma^{\tau} u, \sigma^{\tau} v, \sigma^{\tau} x\right)<2 \varepsilon .
\end{aligned}
$$

We deduce

$$
\begin{aligned}
0 & \leq L(p, \mu)-J(u, v, x) \\
& =L_{1}\left(p, \mu_{1}\right)-J(u, v, x)+L(p, \mu)-L_{1}\left(p, \mu_{1}\right) \\
& \leq 2 \varepsilon+L(p, \mu)-L_{1}\left(p, \mu_{1}\right) .
\end{aligned}
$$

Using the limit $\varepsilon \rightarrow 0$ we conclude $L(p, \mu) \geq L_{1}\left(p, \mu_{1}\right)$.

Proof of Theorem III.1. Let $p \in X$ and $\mu^{*}$ be an optimal controller for $\Pi$. By Proposition III. 1 it is enough to verify

$$
L\left(p, \mu^{*}\right) \geq L_{1}\left(p, \mu_{1}\right) .
$$

To this end, let $\bar{\mu}^{*} \in \mathcal{F}(X, U)$ be such that its image coincides with $\mu^{*}$ in the first component but $\bar{\mu}^{*}\left(\left.a\right|_{[0 ; t]},\left.b\right|_{[0 ; t]}\right)_{2}=\{1\}$ if $a(t) \in A_{1}$ for any $a, b$ and $t$. Let $\varepsilon>0$ and $(u, \bar{v}, x) \in$ $\mathcal{B}_{p}\left(\bar{\mu}^{*} \times S\right)$, where $S=(X, U, F)$, satisfy

$$
\left|L_{1}\left(p, \bar{\mu}^{*}\right)-J_{1}(u, \bar{v}, x)\right|<\varepsilon .
$$

Let $\tau=\min \bar{v}^{-1}(1)$ and $q=x(\tau)$. Then $L_{2}\left(q, \mu_{2}\right)=L\left(q, \mu^{*}\right)$ by the optimality of $\mu_{2}$ and as $q \in A_{1}$. So, without loss of generality $\left|L_{2}\left(q, \mu_{2}\right)-J\left(\sigma^{\tau} u, \sigma^{\tau} v, \sigma^{\tau} x\right)\right|<\varepsilon$, where $v \in\{0,1\}^{\mathbb{Z}_{+}}$ satisfies $v(\tau+t) \in \mu^{*}\left(\left.\left(\sigma^{\tau} x\right)\right|_{[0 ; t]},\left.\left(\sigma^{\tau} u\right)\right|_{[0 ; t]}\right)_{2}$ for $t \in \mathbb{Z}_{+}$and $v([0 ; \tau[)=\{0\}$. It follows

$$
\left|J_{1}(u, \bar{v}, x)-J(u, v, x)\right|<\varepsilon .
$$

By the triangle inequality applied to (16) and (17) it follows

$$
\left|L_{1}\left(p, \bar{\mu}^{*}\right)-J(u, v, x)\right|<2 \varepsilon .
$$

Since $L_{1}\left(p, \mu_{1}\right) \leq L_{1}\left(p, \bar{\mu}^{*}\right)$ by the optimality of $\mu_{1}$ the combination with (18) and the definition of $v$ yields

$$
L_{1}\left(p, \mu_{1}\right) \leq J(u, v, x)+2 \varepsilon \leq L\left(p, \mu^{*}\right)+2 \varepsilon .
$$

Using the limit $\varepsilon \rightarrow 0$ we conclude (15). 


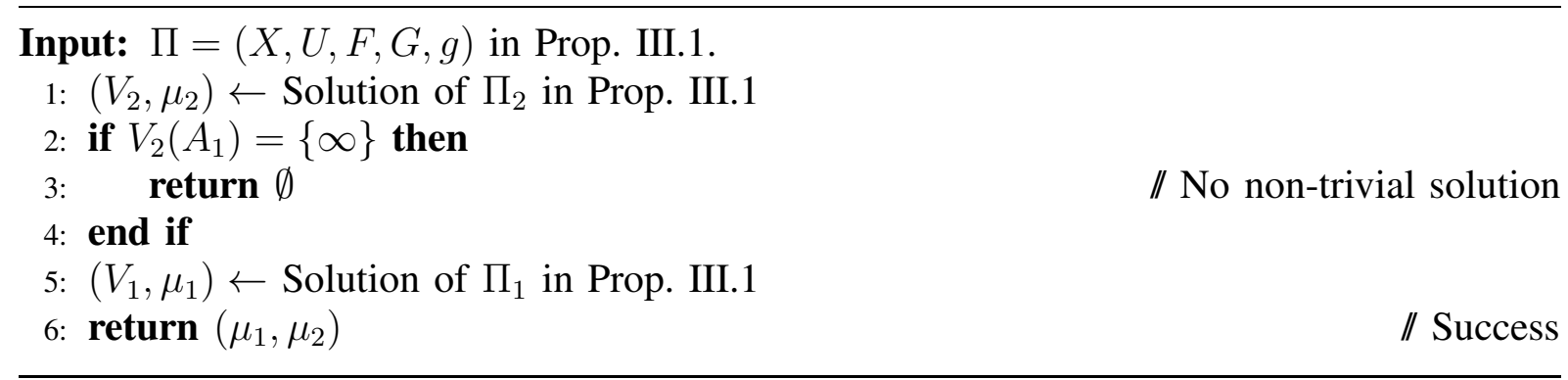

Fig. 2: Procedure to synthesize $\mu$ in (13) such that $\mu$ is optimal for the quantitative two-phase reach-avoid problem $\Pi$.

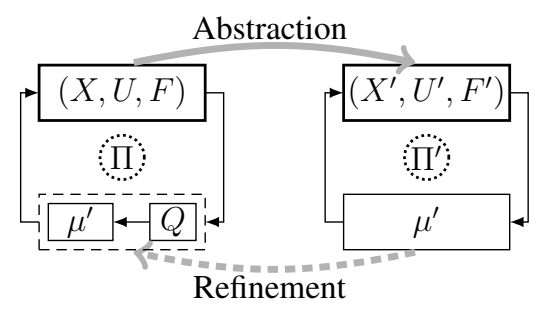

Fig. 3: Principle of symbolic (or abstraction-based) controller synthesis.

\section{Fully-automated approximate solution}

The findings presented previously do not directly imply a constructive method for obtaining an optimal controller (13) since optimal controllers $\mu_{1}$ and $\mu_{2}$ need to be known. Below, a constructive method to approximately solve quantitative two-phase reach-avoid problems on sampled versions of plants governed by the differential inclusion (1) is presented.

To begin with, from Proposition III.1 and Theorem III.1 the synthesis procedure given in Fig. 2 can be deduced, which on success results in an optimal controller for the input problem. In the case that $X$ and $U$ of the input data in Fig. 2 are finite sets, concrete algorithms to perform lines 1 and 5 have been formulated in [8], [14] (based on Dijkstra's algorithm) and [15] (based on the Bellman-Ford algorithm). These concrete algorithms have been developed within the already mentioned framework of Symbolic Optimal Control (SOC) [8] and whose motivations were to efficiently solve optimal control problems with terminal cost (rather than trajectory cost) on sampled-data plants.

In what follows, we show how to utilize SOC to deduce the announced constructive method. We begin with a brief review on the synthesis method of SOC. The key idea is to lift the original optimal control problem $\Pi=(X, U, F, G, g)$ to an abstract optimal control problem $\Pi^{\prime}$ with finite state space $X^{\prime}$ and finite input set $U^{\prime}$. In fact, $X^{\prime}$ is chosen as a finite cover of $X$ and $U^{\prime}$ as a subset of $U$. The fundamental results of symbolic (or abstractionbased) controller synthesis then allow to conclude an approximate solution for the original problem $\Pi$ (in case of a terminal cost (5)) by controller refinement. Specifically, the refined controller is the interconnection of the "abstract" controller $\mu^{\prime}$ synthesized for $\Pi^{\prime}$ and a quantizer ("A/D converter")

$$
Q: X \rightrightarrows X^{\prime}, \quad \Omega \in Q(p): \Leftrightarrow p \in \Omega .
$$

See Fig. 3. The precise statement is as follows [8, Th. V.6, Prop. VI.2].

III.2 Theorem ([8]). Let

$$
\Pi=(X, U, F, G, g) \text { and } \Pi^{\prime}=\left(X^{\prime}, U^{\prime}, F^{\prime}, G^{\prime}, g^{\prime}\right)
$$

be optimal control problems such that $G: X \rightarrow \mathbb{R}_{+} \cup\{\infty\}, G^{\prime}: X^{\prime} \rightarrow \mathbb{R}_{+} \cup\{\infty\}$ and the following holds: 
(i) $X^{\prime}$ is a cover of $X$ by non-empty sets;

(ii) $U^{\prime} \subseteq U$,

and whenever $x \in \Omega \in X^{\prime}, x^{\prime} \in \Omega^{\prime} \in X^{\prime}, u \in U^{\prime}$ :

(iii) $\Omega^{\prime} \cap F(\Omega, u) \neq \emptyset \Rightarrow \Omega^{\prime} \in F^{\prime}(\Omega, u)$;

(iv) $G(x) \leq G^{\prime}(\Omega)$;

(v) $g\left(x, x^{\prime}, u\right) \leq g^{\prime}\left(\Omega, \Omega^{\prime}, u\right)$.

Let $\mu^{\prime} \in \mathcal{F}_{0}\left(X^{\prime}, U^{\prime}\right)$ and let $Q$ be as in (19). Then

$$
L\left(p, \mu^{\prime} \circ Q\right) \leq \sup \left\{L^{\prime}\left(\Omega, \mu^{\prime}\right) \mid p \in \Omega\right\}
$$

for all $p \in X$, where $L$ and $L^{\prime}$ denote the closed-loop performance of $\Pi$ and $\Pi^{\prime}$ respectively.

Furthermore, the results of [8] provide a statement on the distance of $L$ to $L^{\prime}$ as well as a convergence statement. A computational technique to efficiently implement (iii) under certain assumptions on the involved transition system $(X, U, F)$ is given in [4], [5].

Having previous results at hand, the synthesis technique to solve two-phase reach-avoid problems on sampled systems is as given in Fig. 4. Loosely speaking, the idea is to apply Fig. 2 to the abstract problem $\Pi^{\prime}$ and to follow the method depicted in Fig. 3. This results in an approximately optimal controller, whose structure is depicted in Fig. 5 and whose formal properties are provided by next theorem.

Input: Two-phase reach-avoid problem $(X, U, F, G, g)$ associated with $A_{1}, A_{2}$ and $G_{0}$

1: Define $X^{\prime}, U^{\prime}, F^{\prime}$ satisfying (i)-(iii) of Theorem III.2.

2: Define $G^{\prime}$ by (12) with

$$
\begin{aligned}
& A_{i}^{\prime}=\left\{\Omega \in X^{\prime} \mid \Omega \subseteq A_{i}\right\}, i \in\{1,2\} \\
& G_{0}^{\prime}(\Omega) \geq \sup _{x \in \Omega} G_{0}(x) .
\end{aligned}
$$

in place of $A_{1}, A_{2}$ and $G_{0}$, respectively.

3: Define $g^{\prime}$ satisfying (v) in Theorem III.2.

4: Apply the procedure in Fig. 2 to $\left(X^{\prime}, U^{\prime}, F^{\prime}, G^{\prime}, g^{\prime}\right)$

5: if line 4 returns a non-trivial solution then

6: $\quad$ Define $Q$ by (19)

7: $\quad$ Define $\mu_{Q} \in \mathcal{F}(X, U)$ by (13) with $\mu_{1}^{\prime} \circ Q$ and $\mu_{2}^{\prime} \circ Q$

in place of $\mu_{1}$ and $\mu_{2}$, respectively, where $\left(\mu_{1}^{\prime}, \mu_{2}^{\prime}\right)$

is the return value of line 4 .

8: $\quad$ return $\mu_{Q}$

// Success

else

10: $\quad$ return $\emptyset$

1: end if

return $\emptyset$ // Synthesis failed

Fig. 4: Procedure to synthesize an approximately optimal controller for the quantitative two-phase reach-avoid problem $(X, U, F, G, g)$.

III.3 Theorem. Consider the algorithm in Fig. 4 with input $\Pi=(X, U, F, G, g)$ and assume that it returns the controller $\mu_{Q}$. Then $\mu_{Q}$ approximately solves $\Pi$ in the sense that

$$
L\left(p, \mu_{Q}\right) \leq \sup \left\{V^{\prime}(\Omega) \mid p \in \Omega\right\}
$$

for all $p \in X$, where $L$ denotes the closed-loop performance of $\Pi$ and $V^{\prime}$ stands for the value function of the abstract optimal control problem $\Pi^{\prime}=\left(X^{\prime}, U^{\prime}, F^{\prime}, G^{\prime}, g^{\prime}\right)$ in line 4. 


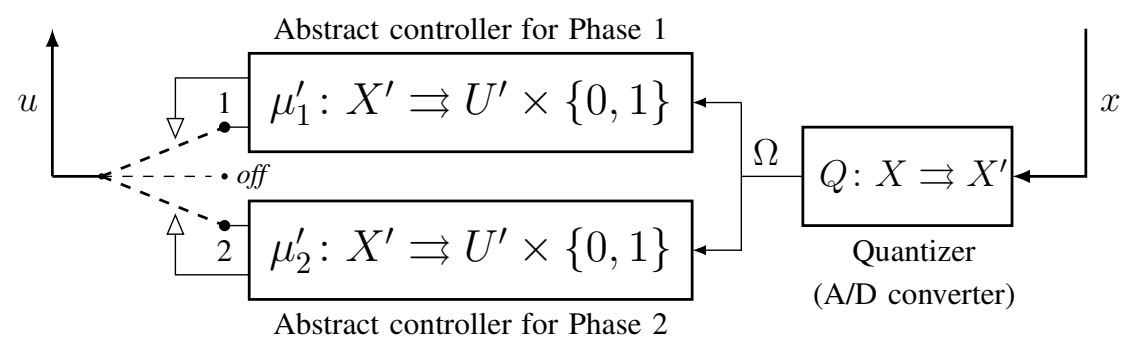

Fig. 5: Approximately optimal controller for quantitative two-phase reach-avoid problems on sampled-data systems. The switch moves in the order "1-2-off" according (13), where "off" occurs when $\mu_{2}^{\prime}$ signals stopping.

Proof. Let $\Pi_{2}, L_{1}, L_{2}$ be as in Proposition III.1 when applied to $\Pi$ with $\mu_{i}:=\mu_{i}^{\prime} \circ Q$, $i \in\{1,2\}$. Firstly, for $p \in X$ it follows that $L\left(p, \mu_{Q}\right)=L_{1}\left(p, \mu_{1}\right)$. Secondly, fix $\varepsilon>0$. There exists $(u, v, x) \in \mathcal{B}_{p}\left(\mu_{1} \times S\right)$, where $S=(X, U, F)$, such that for $\tau=\min v^{-1}(1)$ the value $L_{1}\left(p, \mu_{1}\right)$ is less than

$$
\sum_{t=0}^{\tau-1} g(x(t), x(t+1), u(t))+L_{2}\left(x(\tau), \mu_{2}\right)+\varepsilon .
$$

Next, by [4, Th. V.4] there exists $\Omega \in\left(X^{\prime}\right)^{\mathbb{Z}_{+}}$satisfying a) $(u, v, \Omega) \in \mathcal{B}_{\Omega(0)}\left(\mu_{1}^{\prime} \times S^{\prime}\right)$, b) $x(t) \in \Omega(t)$ for all $t \in \mathbb{Z}_{+}$and c) $L_{2}\left(x(\tau), \mu_{2}\right) \leq L_{2}^{\prime}\left(\Omega(\tau), \mu_{2}^{\prime}\right)$, where $S^{\prime}=\left(X^{\prime}, U^{\prime}, F^{\prime}\right)$ and $L_{2}^{\prime}$ denotes the closed-loop performance of the optimal control problem $\Pi_{2}^{\prime}$ defined in line 1 of Fig. 2 when applied to $\Pi^{\prime}$. Property c) follows from Theorem III.2 applied to $\Pi_{2}$ and $\Pi_{2}^{\prime}$. Finally, apply Proposition III. 1 to $\Pi^{\prime}, \mu_{1}^{\prime}, \mu_{2}^{\prime}$ in place of $\Pi, \mu_{1}, \mu_{2}$ and denote by $L_{1}^{\prime}$ and $L_{2}^{\prime}$ the closed-loop performances appearing Prop. III.1 for this case. By Theorem III.2 and properties b), c) above, (21) is bounded by

$$
\sum_{t=0}^{\tau-1} g^{\prime}(\Omega(t), \Omega(t+1), u(t))+L_{2}^{\prime}\left(\Omega(\tau), \mu_{2}^{\prime}\right)+\varepsilon .
$$

The latter sum is less than $L_{1}^{\prime}\left(\Omega(0), \mu_{1}^{\prime}\right)+\varepsilon$ by property a) above. Apply Theorem III.1 to $\Pi^{\prime}$ and $\mu_{1}^{\prime}, \mu_{2}^{\prime}$ to see that the last sum equals $V^{\prime}(\Omega(0))+\varepsilon$. It follows $L_{1}\left(p, \mu_{1}\right) \leq V^{\prime}(\Omega(0))$ by letting $\varepsilon \rightarrow 0$, which implies (20).

\section{EXAMPLES}

The theoretical results are illustrated by experimental evaluations on two examples. In the first, the example already appeared in Section I will be discussed in detail while in the second a multiple application of Fig. 4 is considered.

\section{A. Urban parcel delivery with vehicle model}

1) Control problem: A variant of the classical vehicle model [24, Ch. 2.4] is considered, whose dynamics is described by (1) with $W=\{(0,0)\} \times\left[-\frac{1}{100}, \frac{1}{100}\right] \times\left[-\frac{1}{10}, \frac{1}{10}\right]$,

$$
f\left(\left(x_{1}, \ldots, x_{4}\right),\left(u_{1}, u_{2}\right)\right)=\left(\begin{array}{c}
x_{4} \cdot \cos \left(\alpha+x_{3}\right) \cdot \beta \\
x_{4} \cdot \sin \left(\alpha+x_{3}\right) \cdot \beta \\
x_{4} \cdot \tan \left(u_{2}\right) \\
u_{1}
\end{array}\right)
$$

where $\alpha=\arctan \left(\tan \left(u_{2}\right) / 2\right), \beta=\cos (\alpha)^{-1}$. Here, $\left(x_{1}, x_{2}\right)$ describes the position, and $x_{3}$ and $x_{4}$ describe the orientation and the velocity of the vehicle, respectively. The control inputs $u_{1}$ and $u_{2}$ are the acceleration and the steering angle of the vehicle, respectively. Formally, 


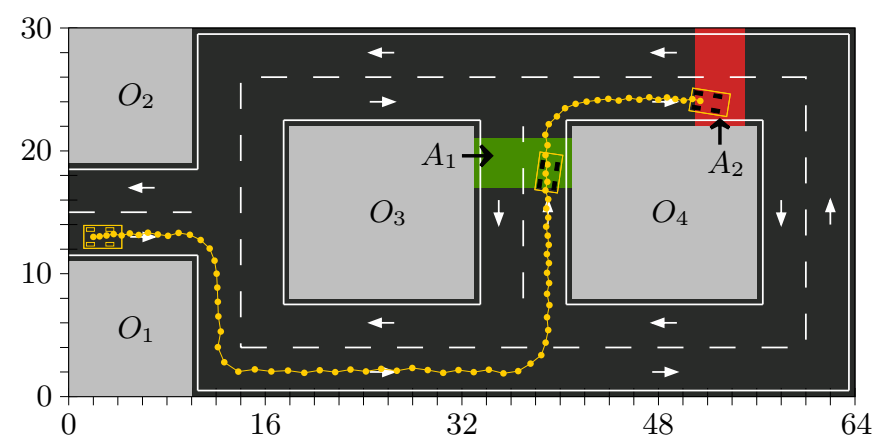

Fig. 6: Illustration of a closed-loop trajectory as obtained from interconnecting the plant in Section IV-A with an approximately optimal controller for the given quantitative two-phase reach-avoid problem. The vehicle symbols indicate the initial state $(2,13,0,5)$ and the states where switching of controllers occurs (cf. Fig 5), respectively.

we consider the system $\left(\mathbb{R}^{4}, U, F\right)$ defined as the sampled system [4, Def. VIII.1] associated with (1) and sampling time $\tau=0.1$, where $f$ is given by (23), $U=[-6,4] \times[-0.5,0.5]$.

The vehicle is located in the urban environment shown in Fig. 1. We are going to solve the quantitative two-phase reach-avoid problem $\Pi$ of the form (7) associated with $A_{1}, A_{2}$ and $G_{0}$, where $G_{0}$ is the zero function and

$$
\begin{aligned}
& A_{1}=[33,41] \times[17,21] \times[-\pi, \pi] \times[0,7], \quad \text { (“Area 1") } \\
& A_{2}=[51,55] \times[22,30] \times[-\pi, \pi] \times[0,7] . \quad \text { (“Area 2") }
\end{aligned}
$$

The running cost $g$ satisfies $g(x, y, u)=\infty$ if $x$ violates the common right-hand traffic rules or is in the obstacle set $\left(\mathbb{R}^{4} \backslash \bar{X}\right) \cup O_{1} \cup \ldots \cup O_{4}$ which restricts space and velocity. Here,

$$
\begin{aligned}
\bar{X} & =[0,64] \times[0,30] \times[-\pi, \pi] \times[0,18], \\
O_{1} & =[0,10] \times[0,11] \times[-\pi, \pi] \times[0,18], \\
O_{3} & =[18,33] \times[8,22] \times[-\pi, \pi] \times[0,18]
\end{aligned}
$$

and $O_{2}=(0,19,0,0)+O_{1}, O_{4}=(23,0,0,0)+O_{3}$. The traffic rules are not violated if, e.g., $x$ is in the set

$$
[10,64] \times[0,4] \times[-3 \pi / 8,3 \pi / 8] \times[0,18],
$$

in which case $g$ compromises minimum time and proper driving style by satisfying

$$
g(x, y, u)=\tau+u_{2}^{2}+\min _{m \in M}\left\|\left(y_{1}, y_{2}\right)-m\right\|_{2} .
$$

Here, $\|\cdot\|_{2}$ is the Euclidean metric on $\mathbb{R}^{2}$ and $M \subseteq \mathbb{R}^{2}$ describes the axis of each roadway. E.g. $[12,62] \times\{2\} \subseteq M$.

2) Approximate solution: To solve $\Pi$ approximately, we apply the procedure in Fig. 4 . We use [4, Sect. VIII] in combination with the technique in [15, Sect. IV] to compute a discrete abstraction $\left(X^{\prime}, U^{\prime}, F^{\prime}\right)$ for the sampled system, i.e. to perform line 1 in Fig. 4 and to solve the two abstract optimal control problems, i.e. lines 1 and 5 in Fig. 2 invoked by line 4 in Fig. 4. The synthesis is successful. A closed-loop trajectory is shown in Fig. 6. The cost (6) for the shown trajectory is 31.78 whereas the corresponding cost of the closed-loop trajectory shown in Fig. 1 is $24 \%$ higher (39.50). Some more computational details are given in Tab. I. 


\begin{tabular}{|l|l|}
\hline Quantity & Value (description) \\
\hline \hline$\left|X^{\prime}\right|$ & $\approx 21.2 \cdot 10^{6}$ (subdivision of (24a) into \\
& $120 \cdot 57 \cdot 62 \cdot 50$ hyper-rectangles) \\
$\left|U^{\prime}\right|$ & $99(9 \cdot 11$ values of $U$ ) \\
\hline Runtime line 1 in Fig. 2 & $590 \mathrm{sec}$. \\
Runtime line 5 in Fig. 2 & $412 \mathrm{sec}$. \\
Total runtime (Fig. 4) & $16.7 \mathrm{~min}$. \\
Total RAM usage & $9.9 \mathrm{~GB}$ \\
\hline
\end{tabular}

TABLE I: Computational details to Section IV-A. Runtimes refer to a computation in parallel with 24 cores (Intel Xeon E5-2697@ $2.6 \mathrm{GHz}$ ) on a Linux OS using an implementation of Fig. 4 in C.

\section{B. Routing mission with fixed-wing aircraft model}

Next, the powerfulness of our results is demonstrated by considering a control problem for a fixed-wing aircraft, whose mission is to fly to three "areas of interest" consecutively after departure from the airfield and then back to it. A prescribed cost function is to be minimized. Hence, a more general optimal control problem than in Definition III.1 is considered and we will use the results of Section III formally as a heuristic only. Nevertheless, the result is remarkable.

To anticipate the outcome straightaway, consider Fig. 7: The controller obtained by the results of this work requires for the mission 140 time steps (the cost is 70.34 as clarified later) leading to the multicoloured trajectory in Fig. 7. In contrast, a sequence of "locally" optimal controllers requires 184 time steps (the cost is $36 \%$ higher) leading to the entangled trajectory that is also indicated. The details to the control problem are given below.

1) Control problem: The plant is a four-dimensional fixed-wing aircraft model. The states are the planar position $\left(x_{1}, x_{2}\right)$, heading and velocity $\left(x_{3}\right.$ and $x_{4}$, respectively) of the aircraft. The inputs are the thrust and the bank angle ( $u_{1}$ and $u_{2}$, respectively) of the aircraft. The function $f$ in (1) is given by $f_{1}$ in [15, Eq. 14], see also [25, Sect. 3]. As in Section IV-A we formally consider the sampled system $\left(\mathbb{R}^{4}, U, F\right)$ associated with (1) and sampling time $\tau=0.45$ with $W=\{0\}$, and $U=[0,18000] \times\left[-40^{\circ}, 40^{\circ}\right]$.

The sets defining the mission are given in Tab. II. The trajectory cost (5) is zero if the sets are visited in the order $A_{1}, A_{2}, A_{3}, A_{\text {rwy }}$ while the running cost (4) is given by

$$
g(x, y, u)=\left\{\begin{array}{ll}
\infty, & \text { if } x \in\left(\mathbb{R}^{4} \backslash X_{\text {mis }}\right) \cup O \\
\tau+u_{2}^{2}, & \text { otherwise }\left(u_{2} \text { in radians }\right)
\end{array} .\right.
$$

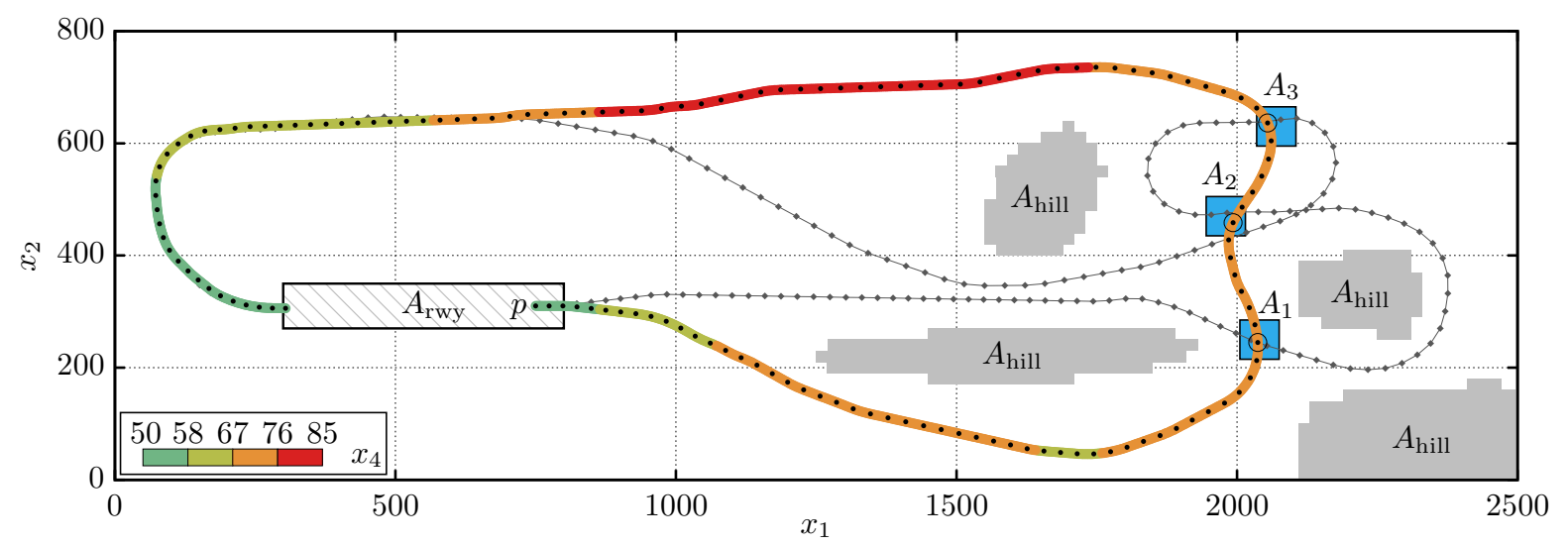

Fig. 7: Routing mission from Section IV-B. Closed-loop trajectories starting at $p=\left(750,310,0^{\circ}, 52.5\right)$ are illustrated, where the multicoloured one is obtained from results of this work while the gray-coloured one is obtained from naively solving the given problem through four classical reach-avoid problems (Definition II.4). At the circled points $(\odot)$ of the multicoloured trajectory the switching to the next controller occurs (cf. Fig. 5). 


\begin{tabular}{|lll|}
\hline Symbol & Value & Meaning \\
\hline \hline$X_{\text {mis }}$ & {$[0,2500] \times[0,800] \times \mathbb{R} \times[50,85]$} & Mission area and admissible heading and velocity \\
\hline$A_{1}$ & {$[2005,2075] \times[215,285] \times \times \mathbb{R} \times[50,75]$} & $1^{\text {st }}$ area of interest and admissible fly-over velocity \\
\hline$A_{2}$ & {$[1945,2015] \times[435,505] \times \mathbb{R} \times[50,75]$} & $2^{\text {nd }}$ area of interest and admissible fly-over velocity \\
\hline$A_{3}$ & {$[2035,2105] \times[595,665] \times \mathbb{R} \times[50,75]$} & $3^{\text {rd }}$ area of interest and admissible fly-over velocity \\
\hline$A_{\text {rwy }}$ & {$[300,800] \times[270,350] \times\left[-10^{\circ}, 10^{\circ}\right] \times[50,55]$} & Runway and admissible heading and velocity for landing \\
\hline$A_{\text {nofly }}$ & {$[320,780] \times[290,330] \times\left[12^{\circ}, 348^{\circ}\right] \times \mathbb{R}$} & Illegal aircraft states over runway \\
\hline$A_{\text {hill }}$ & $\subseteq \mathbb{R}^{2}$, see Fig. 7 & Spatial obstacle set \\
\hline$O$ & $\left(A_{\text {hill }} \times \mathbb{R}^{2}\right) \cup A_{\text {nofly }}$ & Overall obstacle set \\
\hline
\end{tabular}

TABLE II: Sets defining the optimal control problem in Section IV-B.

Thus, the cost to minimize is a compromise between small bank angles and minimum time to accomplish mission.

To solve this mission, it is formalized by two quantitative two-phase reach-avoid problems $\Pi_{1}$ and $\Pi_{2}$ given by (7) with $G_{i}$ in place of $G$ defined as follows:

- $\Pi_{2}$ is associated with $A_{3}, A_{\text {rwy }}$ and the zero function;

- $\Pi_{1}$ is associated with $A_{1}, A_{2}$ and $V_{2}$, where $V_{2}$ is the value function of $\Pi_{2}$.

2) Approximate solution: For the approximate solution we use two abstract optimal control problems $\left(X^{\prime}, U^{\prime}, F^{\prime}, G_{i}^{\prime}, g^{\prime}\right), i \in\{1,2\}$, where the discrete abstraction (line 1 in Fig. 4) is computed exactly as in [15, Sect. VI], e.g. $\left|X^{\prime}\right| \approx 141.7 \cdot 10^{6},\left|U^{\prime}\right|=35$. The non-trivial cases in the definition of $G_{1}^{\prime}$ and $G_{2}^{\prime}$ equal the value function of $\Pi_{2}^{\prime}$ and the zero function, respectively. The total runtime and RAM usage for solving $\Pi_{1}^{\prime}$ and $\Pi_{2}^{\prime}$ is 3 hours and 52 GB using the technique in [15] (hardware as in Section IV-A).

\section{CONCLusions AND OUtLOOK}

From the theoretical point of view this work provides the structure of an optimal controller for solving two-phase reach-avoid problems on time-discrete nonlinear systems. In addition, a method to synthesize approximately optimal controllers on sampled-data systems has been deduced.

Practicality of our results has been proved by simulations. In particular, our theoretical contributions proved to be useful also as a heuristic to solve approximately optimal control problems where several target sets must be visited.

Many open questions remain. Obviously, the structure of an optimal controller for several target sets is of interest as a generalization of Proposition III.1 and Theorem III.1. Another open question is if a necessary condition for the optimality of such controllers can be formulated. An even more general open problem is to generalize Theorem III.2 to optimal control problems with trajectory cost (rather than terminal cost).

\section{REFERENCES}

[1] Y. Liu, Y. Peng, B. Wang, S. Yao, and Z. Liu, "Review on cyber-physical systems," IEEE/CAA Journal of Automatica Sinica, vol. 4, no. 1, pp. 27-40, Jan 2017.

[2] P. Tabuada, Verification and control of hybrid systems. New York: Springer, 2009.

[3] A.-K. Schmuck, Building bridges in abstraction-based controller synthesis. epubli GmbH, 2015, Dissertation.

[4] G. Reissig, A. Weber, and M. Rungger, "Feedback refinement relations for the synthesis of symbolic controllers," IEEE Trans. Automat. Control, vol. 62, no. 4, pp. 1781-1796, Apr. 2017.

[5] A. Weber, Methoden zur Effizienzsteigerung abstraktionsbasierter Reglerentwurfsverfahren. München: Dr. Hut, 2018, Dissertation.

[6] F. de Roo and M. Mazo, "On symbolic optimal control via approximate simulation relations," in Proc. IEEE Conf. Decision and Control (CDC). New York: IEEE, Dec 2013, pp. 3205-3210.

[7] G. Reissig and M. Rungger, "Abstraction-based solution of optimal stopping problems under uncertainty," in Proc. IEEE Conf. Decision and Control (CDC). New York: IEEE, Dec 2013, pp. 3190-3196.

[8] — "Symbolic optimal control," IEEE Trans. Automat. Control, vol. 64, no. 6, pp. 2224-2239, June 2019.

[9] A. Girard, "Synthesis using approximately bisimilar abstractions: state-feedback controllers for safety specifications," in Proc. 13th ACM Intl. Conf. Hybrid Systems: Computation and Control (HSCC), 2010, pp. 111-120. 
[10] P. Meyer, A. Girard, and E. Witrant, "Compositional abstraction and safety synthesis using overlapping symbolic models," IEEE Trans. Automat. Control, vol. 63, no. 6, pp. 1835-1841, June 2018.

[11] K. Hsu, R. Majumdar, K. Mallik, and A. Schmuck, "Lazy abstraction-based control for safety specifications," in Proc. IEEE Conf. Decision and Control (CDC). New York: IEEE, Dec. 2018, pp. 4902-4907.

[12] M. Mazo and P. Tabuada, "Symbolic approximate time-optimal control," Systems \& Control Letters, vol. 60, no. 4, pp. $256-263,2011$.

[13] M. Khaled, E. S. Kim, M. Arcak, and M. Zamani, "Synthesis of symbolic controllers: A parallelized and sparsityaware approach," in Tools and Algorithms for the Construction and Analysis of Systems, T. Vojnar and L. Zhang, Eds. Springer, 2019, pp. 265-281.

[14] E. Macoveiciuc and G. Reissig, "Memory efficient symbolic solution of quantitative reach-avoid problems," in Proc. American Control Conference (ACC). IEEE, July 2019, pp. 1671-1677.

[15] A. Weber, M. Kreuzer, and A. Knoll, "A generalized Bellman-Ford algorithm for application in symbolic optimal control," in Proc. European Control Conf. (ECC), May 2020, pp. 2007-2014. [Online]. Available: https://arxiv.org/abs/2001.06231

[16] Y. Li and J. Liu, "Robustly complete reach-and-stay control synthesis for switched systems via interval analysis," in Proc. American Control Conference (ACC). IEEE, 2018, pp. 2350-2355.

[17] L. Grüne and O. Junge, "Global optimal control of perturbed systems," J. Optim. Theory and Appl., vol. 136, no. 3, pp. 411-429, 2008.

[18] M. Rungger and O. Stursberg, "On-the-fly model abstraction for controller synthesis," in Proc. Amer. Control Conf. (ACC), 2012, pp. 2645-2650.

[19] A. Eqtami and A. Girard, "Safety control, a quantitative approach," IFAC-PapersOnLine, vol. 51, no. 16, pp. 187 192, 2018, 6th IFAC Conf. on Analysis and Design of Hybrid Systems.

[20] G. E. Fainekos, H. Kress-Gazit, and G. J. Pappas, "Temporal logic motion planning for mobile robots," in Proc. IEEE Intl. Conf. Robotics and Automation (ICRA), 2005, pp. 2032-2037.

[21] G. E. Fainekos, A. Girard, H. Kress-Gazit, and G. J. Pappas, "Temporal logic motion planning for dynamic robots," Automatica J. IFAC, vol. 45, no. 2, pp. 343-352, 2009.

[22] T. W. Hungerford, Algebra. New York: Springer, 1974.

[23] R. T. Rockafellar and R. J.-B. Wets, Variational analysis, ser. Grundlehren der Mathematischen Wissenschaften. Berlin: Springer-Verlag, 1998, vol. 317, 3rd corr printing 2009.

[24] K. J. Åström and R. M. Murray, Feedback systems. Princeton University Press, Princeton, NJ, 2008.

[25] W. Glover and J. Lygeros, "A stochastic hybrid model for air traffic control simulation," in Intl. Workshop on Hybrid Systems: Computation and Control. Springer, 2004, pp. 372-386. 